\title{
THE EQUIVALENT CIRCUITS THÉVENIN AND NORTON
}

\author{
Marius STANILOIU, Horațiu POPESCU, Georgiana REZMERIȚĂ, Mihai IORDACHE \\ Dept. of Electrical Engineering \\ University POLITEHNICA Bucharest, \\ Faculty of Electrical Engineering, Spl. Independentei 313, \\ Bucharest, Romania \\ marius.staniloiu@yahoo.com, horatiu.popescu@hella.com, georgiana.rezmerita@upb.ro, \\ mihai.iordache@upb.ro.
}

\begin{abstract}
The main objectives of this paper are to correctly define the equivalent Thévenin and Norton circuits and to presents the complete and exact proof of Thévenin and Norton theorems. These circuits allow the separation of the linear portions of the electronic circuits from the nonlinear ones and in this way the polarization process of the electronic devices becomes much more efficient. When linear and/or nonlinear analyzed circuits have a small number of nonlinear circuit elements, their analysis, synthesis, and simulation are performed much more efficiently if the nonlinear part of the circuit is separated from the linear one. By determining the equivalent Thévenin or Norton circuits in relation to the load to connected terminals of a system with two or more magnetic coupled coils, that are part of the wireless power transfer systems, optimal load parameters are determined so that the maximum active power is transmitted to the load.
\end{abstract}

Keywords: Thévenin's theorem, Norton's theorem, magnetic coupled coils.

\section{INTRODUCTION}

Analog and mixed signal integrated circuit technology plays a critical and decisive role in communications and signal processing. For example, the rapid growth of CMOS technology has made analog circuit design extremely attractive [1-16].

A major challenge when approaching analog circuits is DC polarization - getting the required operating points via a quick convergence technique; and the problem worsens as the size and complexity of the circuit increase. The analysis could possibly result in the existence of additional DC operating points or the instability of these operating points as a result of positive reactions.

For example, in the SPICE circuit simulator $[1,3,6,8,16]$, nonlinearities are solved using NewtonRaphson iteration methods; occasionally, the main challenge is to get the circuit analysis procedure to resolve in a reasonable number of iterations. To improve convergence, schemes such as the addition of minimum conductance (GMIN), shunt resistors, adjusting the level of tolerance for outputs, and changing the feed step are normally done in a simulator.

Typically, a bad selection of initial conditions or the use of big and uneven computation steps results in the calculation process becoming unstable or even diverging. Another potential source of problem is a fixed topology of the fixed supply circuit during the polarization process. With that kind of a pre-defined set of conditions, the operational points are found automatically over the course of several lengthy repetitions. [1, 2, 4, 5, 9].

The main objectives of this paper are to correctly define the equivalent Thévenin and Norton circuits and to presents the complete and exact proof of Thévenin and Norton theorems. These circuits allow the separation of the linear portions of the electronic circuits from the non-linear ones and in this way the polarization process of the electronic devices becomes much more efficient. When linear and/or nonlinear circuits have a small number of nonlinear circuit elements, their analysis, synthesis, and simulation are performed much more efficiently if the nonlinear part of the circuit is separated from the linear one. This separation can be done very easily using the equivalent Thevenin and Norton circuits, [12-16].

In this paper we define Thévenin and Norton equivalent circuits, specifying the necessary and sufficient conditions for a single port linear circuit to be replaced by a Thévenin or Norton equivalent circuit. $[1,3,10]$.

By determining the equivalent Thévenin or Norton circuits in relation to the load connection 
terminals of a system of two or more magnetic coupled coils, used in the construction of wireless power transfer systems, optimal load parameters are determined so that the active power transmitted to the load is maximum, $[3,16]$.

A significant obstacle in approaching analog circuits is DC polarization - obtaining the desired operating points via a fast convergence procedure; and the problem worsens due to the circuit's increased size and complexity, [11 - 15].

The purpose of this study is to offer a procedure for guided design of polarization. Our strategy separates the linear portions of an analog circuit from the nonlinear ones and takes more control over the nonlinear portions. This separation of the portions (components) of the circuit is achieved by introducing a new port model that cancels the ports of nonlinear devices, [11 - 16].

Section 2 defines Thévenin and Norton equivalent circuits, specifying conditions necessary and sufficient for a single-port linear circuit to be replaced by a Thévenin or Norton equivalent circuit. Section 3 sets out the necessary and sufficient conditions for a single-port linear circuit to be replaced by an equivalent hybrid circuit.

Thévenin, Norton are used in the construction of Nullified equivalent circuits. Equivalent Thévenin, Norton, circuits are used in the local polarization of analog circuits, [1 - 16]. Several illustrative examples are presented that highlight the certainty of the elaborated procedures.

\section{THE CIRCUITS EQUIVALENT THÉVENIN AND NORTON}

The circuits equivalent Thévenin and Norton, alternatively referred to as the Thévenin and Norton models, are two well-known models that describe the terminal behavior of linear circuits They are critical in circuit analysis because they serve as a substitute for a terminal port by providing an independent source and an impedance [1, 2, $11-16]$. Both the Thévenin and Norton models are very valuable circuit simplification approaches that are often used to isolate the terminal behavior of particular linear components of the circuit that ordinarily provide energy and signal the remainder of the circuit. In general, these models are used to analyze and apply analog circuits in a variety of ways, including source transformation, analysis in DC, and analysis in AC (frequency or phasor and s-domain) [1, $2,4,9]$.

Definition 1: A linear one-port $N$ (operating in: direct current - DC, alternating current - AC in complex or operational - when using the Laplace transform, with zero initial conditions) is said to be well-defined if and only if it does not contain any circuit elements that are electrically or non-electrically coupled to certain variables external to it $N$ (to some physical variable outside of $N$ ): e.g., controlled sources that depend on variables outside of $N$, windings of transformers magnetically coupled with windings outside of $N$, a photoresistor coupled with an external light source, etc. [9-11].

\subsection{Thévenin $N_{e q}$ equivalent uni-port circuit}

For simplicity of presentation, a resistive linear bipolar circuit (two-port $N$ ), which contains both independent and controlled sources is shown in Fig. 1. Everything that will be exposed in the following are valid also for the two-port linear circuits that work in harmonic regime in complex (AC electrical circuits) and in operational (when using the Laplace transform) with null initial conditions.

The unique solvency condition of the Thévenin $N_{e q}$ equivalent uni-port circuit: A resistive linear circuit $N$ (Fig. 1, a) in which the resistor $R_{A B}$ is replaced by a source independent of current $\mathrm{j}$ (oriented from node $\mathrm{B}$ to node $\mathrm{A}$ ) has a unique solution for $V_{A B}$ voltage for any numerical value of current $\mathrm{j},[9,16]$.

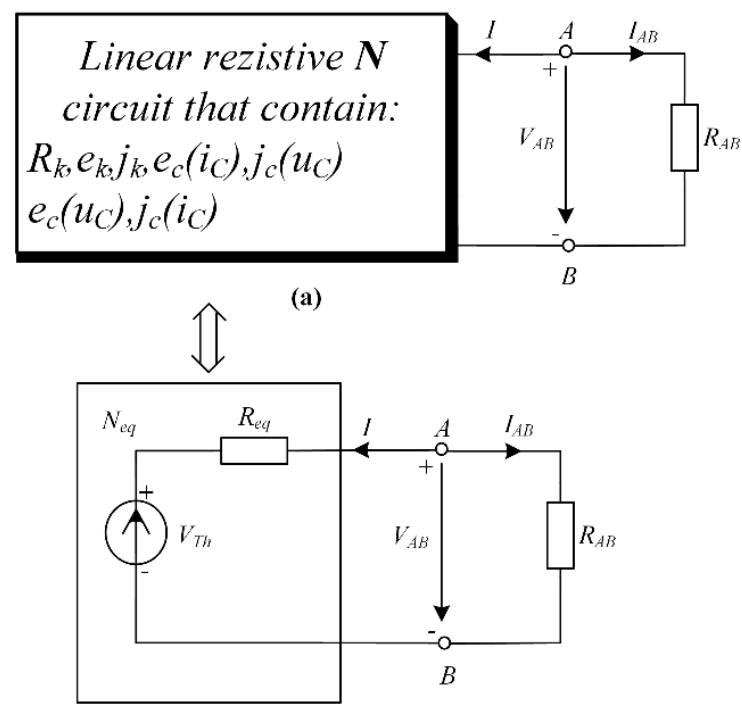

(b)

Fig. 1. a) Diagram of a linear resistive twoport circuit; b) Thévenin equivalent circuit.

If the circuit $N$ in Fig. 1, satisfies the unique solvency condition above, then the circuit $N$ can be substituted with the equivalent voltage generator with $E_{e}=V_{T h}=V_{A B O}$ and $R_{i}=R_{e q}=R_{A B O} \quad\left(E_{e}=V_{T h}=V_{A B O}\right.$ and $Z_{i}=Z_{e q}=Z_{A B O} \quad$ - for AC circuits and $E_{e}(s)=V_{T h}(\mathrm{~s})=V_{A B O}(s)$ and $Z_{i}(s)=Z_{e q}(s)=Z_{A B O}(s)$ - in the case of circuits in operational with zero initial 
conditions, called Thévenin equivalent one-port $N_{e q}$ (Fig. 1, b). If the $R_{A B}$ resistance, the $Z_{A B}$ complex impedance and the $Z_{A B}(s)$ operational impedance are connected between terminals $\mathrm{A}, \mathrm{B}$, then the currents $I_{A B}, I_{A B}$ and respectively $I_{A B}(s)$ have the following expressions:

$$
\begin{gathered}
I_{A B}=\frac{V_{T h}}{R_{e q}+R_{A B}} ; \\
I_{A B}=\frac{\underline{V}_{T h}}{\underline{Z}_{e q}+\underline{Z}_{A B}} \text { and } \\
I_{A B}(s)=\frac{V_{T h}(s)}{Z_{e q}(s)+Z_{A B}(s)},
\end{gathered}
$$

where: $R_{e q}=R_{A B 0}$ is the equivalent resistance, in relation to terminals $\mathrm{A}, \mathrm{B}$, when $R_{A B} \rightarrow \infty$ (driving point or input resistance), $\underline{Z}_{e q}=\underline{Z}_{A B 0}$ represents the equivalent complex impedance, in relation to terminals A, B, when $\underline{Z}_{A B} \rightarrow \infty$ (driving point or input complex impedance), $Z_{e q}(s)=Z_{A B 0}(s)$ - represents the equivalent operational impedance, in relation to terminals $\mathrm{A}, \mathrm{B}$, when $Z_{A B}(s) \rightarrow \infty$ (driving point or input operational impedance) of the passivated circuit $\mathrm{N}$ (after all independent sources inside $\mathrm{N}$ are set to zero, $e_{k}=0$ and $j_{k}=0$ for any $k$ ) - called: Thévenin equivalent resistance, Thévenin equivalent complex impedance and Thévenin equivalent operational impedance. $V_{T h}=V_{A B O}$ - is the $V_{A B}$ open-circuit voltage $\left(R_{A B} \rightarrow \infty\right), \underline{V}_{T h}=\underline{V}_{A B 0}-$ is the $\underline{V}_{A B}$ open-circuit complex voltage $\left(Z_{A B} \rightarrow \infty\right)$ and $V_{T h}(s)=V_{A B 0}(s)$ represents the $\mathrm{VAB}(\mathrm{s})$ open-circuit operational voltage $\left(Z_{A B}(s) \rightarrow \infty\right)$, of the active circuit in Fig. 1, a (open-circuit voltages are voltages $V_{A B}, \underline{V}_{A B}$ and $V_{A B}(s)$ across terminals $\mathrm{A}$ and $\mathrm{B}$ when the port $\mathrm{A}, \mathrm{B}$ is left open-circuited).

\subsection{Norton $N_{e q}$ equivalent uni-port circuit}

Again, for simplicity of exposure, a resistive linear bipolar circuit (two-port $N$ ), which contains both independent and controlled sources is shown in Fig. 1. Everything that will be exposed in the following are valid also for the two-port linear circuits that work in harmonic regime in complex (AC electrical circuits) and in operational (when using the Laplace transform) with null initial conditions.

Unique solvability condition for Norton equivalent one port $N_{e q}$ : A resistive linear circuit $\mathrm{N}$ (Fig. 2, a) in which the $G_{A B}$ conductance is replaced by an independent voltage source e (oriented from node $\mathrm{B}$ to node A) has a unique solution for $I_{A B}$ current for any numeric value of electromotive voltage. [1-3, 1116].

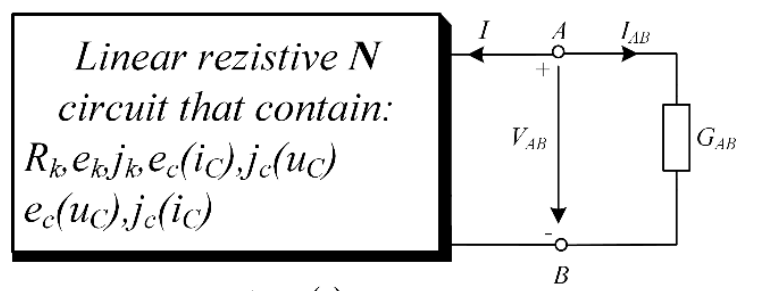

(a)

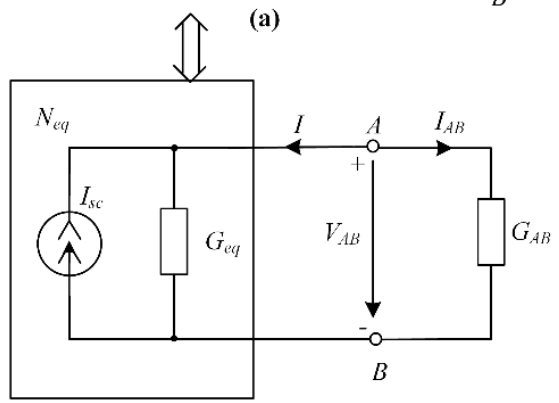

(b)

Fig. 2. a) Diagram of a linear resistive two-port circuit; b) Norton equivalent circuit.

If the circuit $N$ in Fig. 2, a satisfies the unique solvency condition above, then the circuit $N$ can be substituted with the equivalent current generator with $J_{e}=-I_{s c}=I_{A B s c}$ and $G_{i}=G_{e q}\left(\underline{J}_{e}=-\underline{I}_{s c}=\underline{I}_{A b s c}\right.$ and $\underline{Y}_{i}=$ $\underline{Y}_{e q}$ - for AC circuits and $J_{e}(s)=-I_{s c}(s)=I_{A b s c}(s)$ and $Y_{i}(s)=Y_{e q}(s)$ - in the case of circuits in operational with zero initial conditions called Norton equivalent oneport $N_{e q}$ (Fig. 2, b). If the $G_{A B}$ conductance, the $\underline{Y}_{A B}$ complex admittance and the $Y_{A B}(s)$ operational admittance are connected between terminals $\mathrm{A}, \mathrm{B}$, then the currents $V_{A B}, \underline{V}_{A B}$ and respectively $V_{A B}(s)$ have the following expressions:

$$
\begin{gathered}
V_{A B}=\frac{I_{s c}}{G_{e q}+G_{A B}}, \\
V_{A B}=\frac{I_{A B s c}}{\underline{Y}_{e q}+\underline{Y}_{A B}} \text { and } \\
V_{A B}(s)=\frac{I_{A B s c}(s)}{Y_{e q}(s)+Y_{A B}(s)},
\end{gathered}
$$

where: $G_{e q}=G_{A B O}$ is driving point or input conductance across $N$, when $G_{A B} \rightarrow 0, Y_{e q}=Y_{A B 0}$ represents driving point or input admittance across $N$, when $\underline{Y}_{A B} \rightarrow 0, Y_{e q}(\mathrm{~s})=Y_{A B O}(\mathrm{~s})-$ is driving point or input operational admittance across $N$, when $Y_{A B}(s) \rightarrow 0$, after all independent sources inside $N$ are set to zero $\left(e_{k}=0\right.$ and $j_{k}=0$ for any $k$ ) - called: Norton equivalent conductance, Norton equivalent complex admittance and Norton equivalent operational admittance.

$I_{A B s c}=-I_{S c}-$ is the short-circuit current (when $V_{A B}=0$ ), $I_{A B s c}=-I_{S c}-$ represents the short-circuit complex current (when $\underline{V}_{A B}=0$ ) and $I_{A B s c}(\mathrm{~s})=I_{s c}(\mathrm{~s})-$ is the short-circuit operational current (when $V_{A B}(\mathrm{~s})=0$ ). 
Before we prove the above theorems, let us consider first some circuit interpretations and applications. Only resistive linear circuits shall be considered, but the interpretations and applications are also valid for the AC linear circuits and for those in operational, with zero initial conditions.

1. The key benefit of Thévenin's theorem, as well as Norton's theorem, is that it enables us to replace any section of a circuit that forms a linear resistive one-port with just two circuit elements without impacting the solution balance of the circuit.

2. Let $R_{e q} \neq 0$. We acquire the current $I$ if we shortcircuit the Thévenin equivalent $N_{e q}$ and solve for it. (see Fig. 1):

$I_{s c}=-I_{A B s c}=-\frac{V_{T h}}{R_{e q}}$, iar $I_{A B s c}=\frac{V_{T h}}{R_{e q}}$.

3. When $R_{e q} \neq 0$ and $G_{e q} \neq 0$, The $N$ has a single port and is compatible with the Thévenin and Norton models. Its driving-point characteristic defined by:

Thevenin parameters: $\left\{\begin{array}{l}R_{e q} \\ V_{A B 0}=V_{T h}\end{array}\right.$ given $\Rightarrow$ Norton parameters: $\left\{\begin{array}{l}G_{e q}=\frac{1}{R_{e q}} \\ I_{s c}=I_{A b s c}=\frac{V_{T h}}{R_{e g}}\end{array}\right.$;

Norton parameters: $\left\{\begin{array}{l}G_{e q} \\ I_{s c}=I_{A B s c}\end{array}\right.$ given

$\Rightarrow$ Thevenin parameters: $\left\{\begin{array}{l}R_{e q}=\frac{1}{G_{e q}} \\ V_{T h}=\frac{I_{s c}}{G_{e q}}\end{array}\right.$.

Characteristics $V_{A B}-I$ and $I-V_{A B}$ respectively have the following forms:

$$
\begin{gathered}
V_{A B}=R_{e q} I+V_{T h}=-R_{e q} I_{A B}+V_{T h} \text { or } \\
I=G_{e q} V_{A B}-I_{S c} .
\end{gathered}
$$

This driving-point characteristic, $I=G_{e q} V_{A B}$ $-I_{s c}$, consists of a straight line with a slope $G_{e q}$ and current intercept $I_{s c}$ in $V_{A B}-I$ plane, as shown in Fig. 3 , a, or with a slope $R_{e q}$ and intercept $V_{T h}$ in the $I-V_{A B}$ plane.

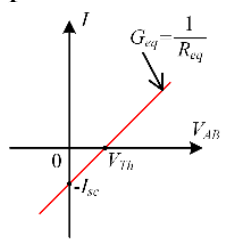

(a)

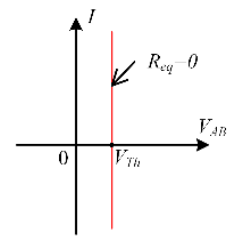

(b)

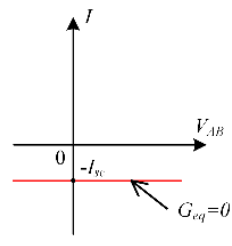

(c)
Fig. 3. a) Characteristic $I-V_{A B}$ of the $N$ two-port with $V_{o s}=V_{T h}>0$ and $G_{e q}>0$; b) Characteristic $I-V_{A B}$ when $V_{A B}=V_{T h}$ and $R_{e q}=0$; c) Characteristic $I-V_{A B}$ when $I=-I_{s c}$ and $G_{e q}=0$.

4. The driving-point characteristic in Fig. 3, a is drawn for the case when $G_{e q}>0, V_{o c}=V_{T h}>0$ and $I_{s c}>0$; in Fig. 3, b displays the limiting scenario when $R_{e q}=0$. In this scenario, the Thévenin equivalent one-port comprises just of a battery with a voltage of $V_{o c}=V_{T h}$ volts. Because $G_{e q}=\infty$, there is no Norton equivalent one-port. Indeed, when a voltage source $V_{A B} \neq V_{o c}$ is applied, the unique solvability criteria fail - KVL is broken. Fig. 3, c presents the "dual" limiting situation $G_{e q}=0$. The Thévenin equivalent oneport does not exist in this case, whereas the Norton equivalent one-port degenerates into a current source of $I_{s c}$ ampers.

5. Fig. 4, a, demonstrates a single port that lacks a Thévenin or Norton equivalent. Its driving-point characteristic is defined by:

$$
V_{A B}=0 \quad I_{A B}=0,
$$

And consists of a single point; essentially, the origin. Important is the "virtual short circuit" that characterizes the input port of an ideal operational amplifier working in the linear zone that has this feature.

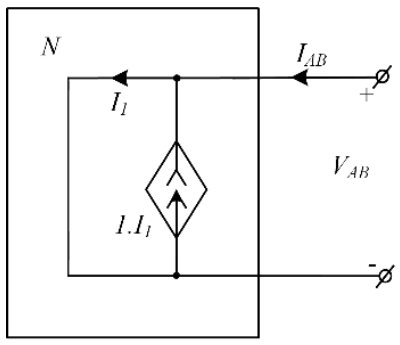

(a)

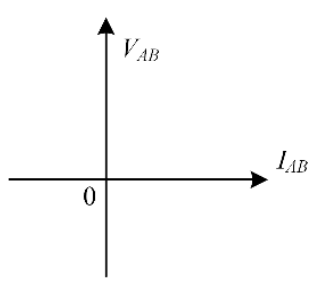

(b)
Fig. 4. Diagram of a one-port characterized by only one point.

Since the driving-point characteristic for both Thévenin and Norton equivalent one-ports consists of a straight line, it is clear the nullator does not have a Thévenin or Norton equivalent one-port. Indeed, both "unique solvability conditions" are violated by this one-port. Note that we can only drive $N$ with a $0-\mathrm{V}$ voltage sources or a $0-\mathrm{A}$ current source.

6. From the above observations if the biport $N$ is not controlled in the current, then this circuit does not possess an equivalent Thévenin circuit. Dual, if $N$ is not voltage controlled, and then the $N$ circuit 
does not have an equivalent Norton circuit. So, in applying Thévenin's or Norton's theorem, one can ignore the verification of the "unique solvency condition" because it generally makes checking the determinant of the associated $\boldsymbol{T}$ matrix array a difficult test. Instead, you can simply calculate $R_{e q}$ or $G_{e q}$. The inability to obtain a unique value for $R_{e q}$ (respectively, $G_{e q}$ ) could imply that the $N$ circuit does not have an equivalent Thévenin circuit (respectively, Norton).

Exemple 1: To determine the equivalent Thévenin and Norton circuits for the bipolar circuit shown in Fig. 5, a. To calculate the parameters $R_{e q}$ and $G_{e q}$ the simplified circuit from Fig. 5, b. For any voltage $V$ applied to terminals $\mathrm{A}, \mathrm{B}$, the current $\mathrm{I}_{1}=V / R$, and from the first Kirchhoff theorem results $I_{l}=-4 I_{l}$. So, $I=-(4 V) / R$. Thus

$$
R_{e q}=\frac{1}{G_{e q}}=-\frac{R}{4} .
$$

Since both $R_{e q}$ and $G_{e q}$ are finite numbers, we can conclude that the circuit $N$ in Fig. 5, a has a one-port circuit (one-port) equivalent to Thévenin and Norton, respectively.

Analyzing the circuits in Fig. 5, c and Fig. 6, a with the ACAP program, [3], is obtained:

$$
\begin{gathered}
V_{T h}=V_{o c}=\frac{4 E+J \cdot R}{4} \text { and } \\
I_{s c}=-\frac{R \cdot J+4 \cdot E}{R} .
\end{gathered}
$$

Therefore $R_{e q}=1 / \mathrm{G}_{\mathrm{eq}}=\mathrm{V}_{\mathrm{Th}} / \mathrm{I}_{\mathrm{sc}}=-\mathrm{R} / 4$. So, the same results are obtained as above.

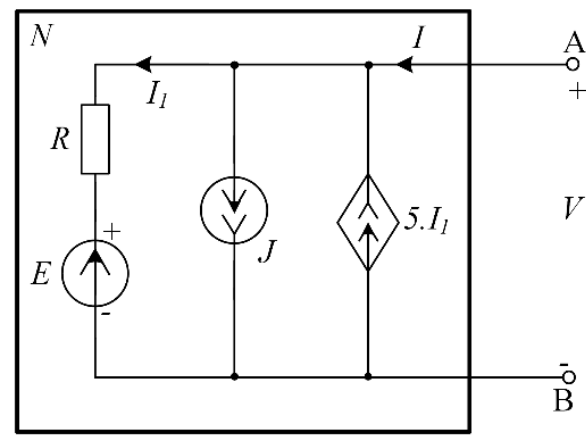

(a)

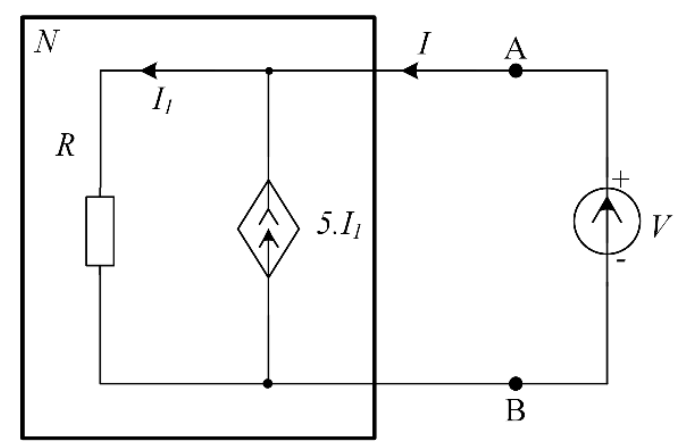

(b)

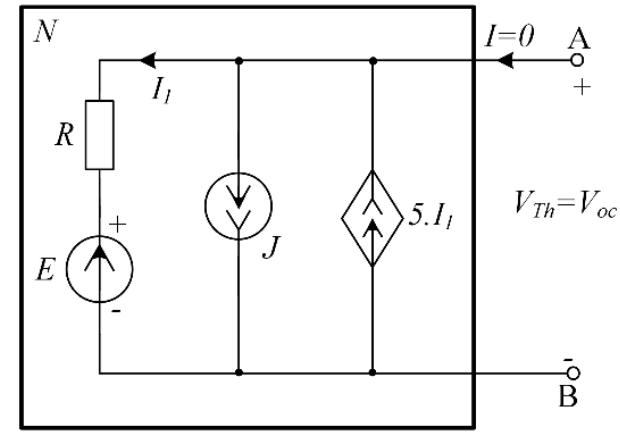

(c)

Fig. 5. a) $\mathrm{N}$ with a single port; b) Setting all independent sources inside $N$ to zero results in a simplified one-port $N$; c) The circuit for computing $V_{T h}$ $=\mathrm{V}_{\mathrm{oc}}$;

Example 1 shows that if the analyzed circuit contains controlled sources, it is possible that the equivalent resistance Thévenin $\mathrm{R}_{\mathrm{eq}}$ or the equivalent conductance Norton $G_{e q}$ can also have negative values.

For the numerical volumes $R=R_{l}=100 \Omega$, $E=E_{2}=100 \mathrm{~V}, \quad J=J_{3}=5 \quad \mathrm{~A}, \quad J_{4}=5 * I_{1}$ and the characteristic $U_{5}-I_{5}$ of the nonlinear resistor $R_{d i 5}$ given by points (see Table 1 ) we obtain:

$R_{T h}=R_{e q}=-25 \Omega, V_{T h}=V_{o c}=225 \mathrm{~V}$, for the equivalent Thévenin circuit.

Table 1 


\begin{tabular}{|l|l|}
\hline $\mathbf{I}_{5}[\mathbf{A}]$ & $\mathbf{V}_{\mathbf{5}}[\mathbf{V}]$ \\
\hline-20 & -200 \\
\hline-10 & -100 \\
\hline-5 & -50 \\
\hline-2 & -20 \\
\hline-1 & -10 \\
\hline 0 & 0 \\
\hline 1 & 10 \\
\hline 2 & 20 \\
\hline 5 & 50 \\
\hline 10 & 100 \\
\hline 20 & 200 \\
\hline
\end{tabular}

Analyzing, with the ACAP program, [3], the circuit in Fig. 6, b results the following values for the voltage and current of the nonlinear resistor $R_{d i 5}, U_{5}=$ $-150 \mathrm{~V}$ and $I_{5}=-15.0 \mathrm{~A}$. If we analyze, with the same ACAP program, [3], the circuit from Fig. 6, c the following results $U_{R d i 5}=-150 \mathrm{~V}$ and $I_{R d i 5}=-15.0 \mathrm{~A}$ are obtained, identical to the above.

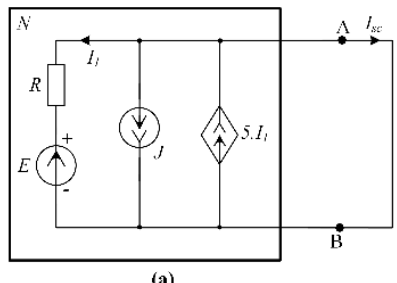

(a)

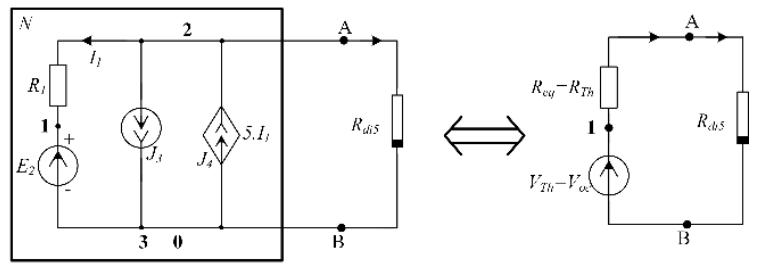

(c)

Fig. 6. a) Circuit used for calculating Isc b); At the terminals A, B of the circuit in Fig. 6, it is connected the current controlled (DC) nonlinear resistor $R_{d i 5}$; c) Circuit $N$ in Fig. 6, b is replaced by the equivalent Thévenin circuit.

2.3 Proof of Thévenin and Norton's theorems

Only Thévenin's theorem will be confirmed since the dual proof will then apply to Norton's theorem. As indicated in Fig. 7, a, let $N$ signify the component in question and $N_{L}$ denote the remainder of the circuit.
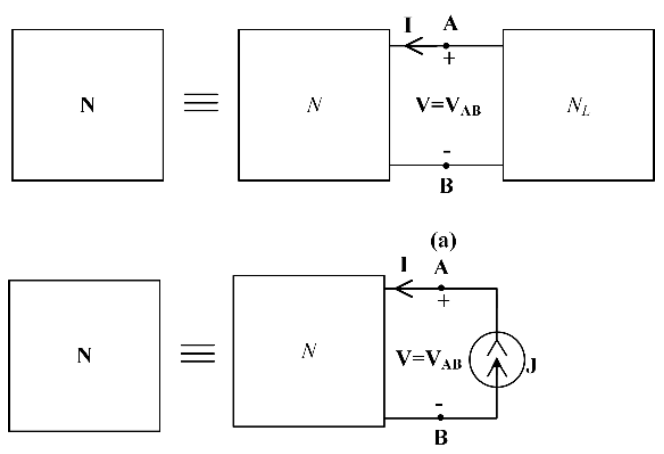

(b)

Fig. 7. a) Partitioning of an arbitrary circuit $N$ into a linear resistive one-port $N$ circuit and another $N_{L}$ circuit that need not be linear or resistive; b) Circuit $N$ is connected to a source independent of current $J$.

Since $N$ is purely resistive, it is completely specified by its driving-point characteristic at each time instant. Therefore, as far as the $N_{L}$ is concerned, its solution depends only on this driving-point characteristic: The elements inside $N$ which give rise to this driving-point characteristic are completely irrelevant. It is sufficient for us to prove that $N$ and its Thévenin equivalent one-port have an identical driving-point characteristic.

Let us drive $N$ with an independent current source $J=I$. It is considered that the circuit $N$ has inside it $n_{E}$ independent voltage sources with electromotive voltage $E_{1}, E_{2}, \ldots, E_{n E}$ and $n_{J}$ sources independent of current $J_{1}, J_{2}, \ldots, J_{n J}$. From the "unique solvency condition" it results that the resistive circuit in Fig. 6, b has a unique solution for all values of independent sources. So, the superposition theorem can be applied and according to this theorem the voltage $V=V_{A B}$ from the terminals A, B of the circuit $N$ has the expression

$$
\begin{aligned}
V= & V_{A B}=R_{A B_{A B}} J \\
& +\sum_{\substack{n_{E} \\
n_{J}}} A_{A B_{k}} E_{k} \\
& +\sum_{k=1}^{n_{A B_{k}} J_{k}},
\end{aligned}
$$

where $R_{A B \_A B}=\left.\frac{V_{A B}}{J}\right|_{\substack{E_{k}=0 \text { for all } k \\ J_{k}=0 \text { for all } k}}=\mathrm{R}_{\mathrm{AB} 0}=\mathrm{R}_{\mathrm{eq}}$ is driving point or input resistance across $N$ after all independent sources inside $N$ are set to zero $\left(E_{k}=0\right.$ ang $J_{k}=0$ for any $k$ ) - called Thévenin equivalent resistance.

$$
A_{A B_{-} k}=\left.\frac{V_{A B}}{E_{k}}\right|_{\begin{array}{c}
E_{m}=0 \text { for all } m \neq k \\
J_{k}=0 \text { for all } k, \text { and } J=0
\end{array}}
$$


represents the voltage transfer factor (voltage gain) from the branch $l_{k}$ to the branch $l_{A B}$, and

$$
R_{A B_{-} k}=\left.\frac{V_{A B}}{J_{k}}\right|_{\substack{E_{k}=0 \text { for all } \\ J_{m}=0 \text { for all } m \neq k, \text { and } J=0}}
$$

is the transfer resistance from the side $l_{k}$ to the side $l_{A B}$.

Now $J=0, \mathrm{~V}$ is, $V=V_{o c}=V_{A B O}=V_{T h}$. Hence, the last two sums from Eq. (9) add up to $V_{T h}$. According to the above arguments the equation (9) can be written in the form.

$$
V=R_{T h} I+V_{T h} \text {. }
$$

Equation (10) gives the driving-point characteristic of the given one-port N. Since this is the same equation which defines the Thévenin equivalent one-port $N_{e q}$, it follows that we can replace $N$ in Fig. 7, a by $N_{e q}$ without affecting the solution inside $N_{L}$.

The Norton theorem can be proved similarly. In this case, the circuit $N$ (fig. 7, b) is supplied with an independent voltage source with electromotive voltage $\mathrm{E}$, after which the superposition theorem is applied and the current $I$ is calculated.

Example 2: In Fig. 8, shows a simplified small signal equivalent circuit of a single stage BJT amplifier with the virtual biasing supplies included. The Thevenin model for the amplifier looking from the output port is given in Fig. 8, b. Fig. 8, c shows the port's characteristic curve (line), indicating the circuit linearity. The Figs. also shows how we can move from the Thévenin model, specified by point $T(2.52 \mathrm{~V}, 0)$, to the Norton model, given as point $N(0,1.26 \mathrm{~mA})$ on the characteristic line.

The circuit to compute the parameters $V_{T h}=V o c$ and $R_{e q}$ the circuit in Fig. 8,d is used, $V_{T h}=$ $V_{o c}=-\left.V_{7}\right|_{J=0 .}=5.52 \mathrm{~V}, I_{S c}=-\left.I_{7}\right|_{V_{7}=0 .}=1.26 \mathrm{~mA}$, $R_{e q}=\frac{V_{T h}}{I_{s c}}=2 \mathrm{k} \Omega$ and $G_{e q}=\frac{1}{R_{e q}}=0.5 \mathrm{mS}$.

To demonstrate that the Thévenin and Norton equivalent circuits are particularly useful in the analysis of nonlinear analog circuits when a nonlinear voltage-controlled $(\mathrm{vc})$ resistor $R_{d u}$ is connected between terminals A, B to the initial circuit - Fig. 8, e, the Thévenin equivalent circuit - Fig. 9, a, and the Norton equivalent circuit - Fig. 9, b, with the nonlinear characteristic $I-U$ given by experimentally determined points in Table 2 .

Table 2

\begin{tabular}{|l|l|}
\hline $\mathbf{V}[\mathbf{V}]$ & $\mathbf{I}[\mathbf{m A}]$ \\
\hline-10 & -10 \\
\hline-5 & -6 \\
\hline-2 & -2.5 \\
\hline-1 & -1 \\
\hline-0.5 & -0.5 \\
\hline 0 & 0 \\
\hline
\end{tabular}

\begin{tabular}{|l|l|}
\hline 0.5 & 10 \\
\hline 1 & 1 \\
\hline 2 & 2.5 \\
\hline 5 & 6 \\
\hline 10 & 10 \\
\hline
\end{tabular}

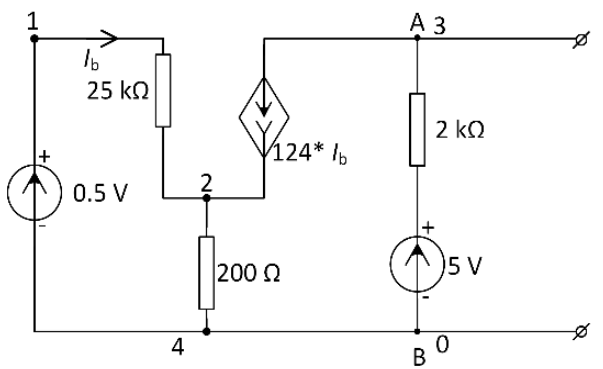

(a)
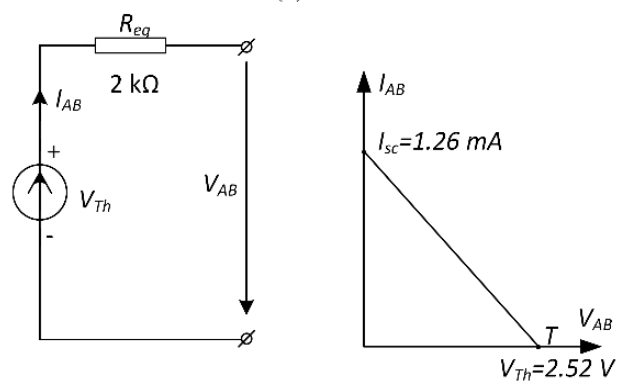

(b)

(c)

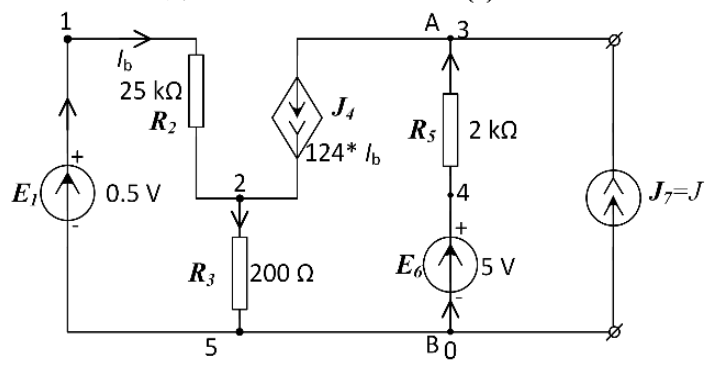

(d)

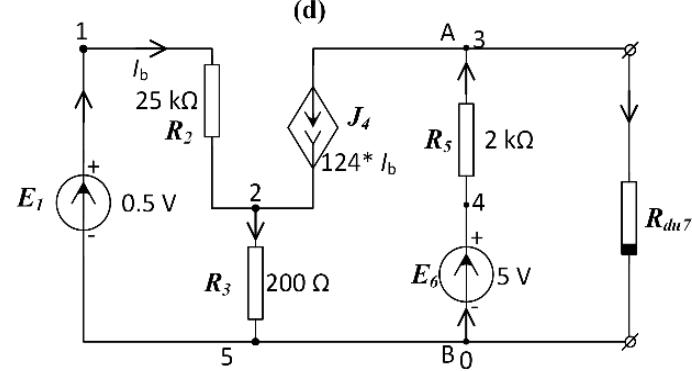

(e)

Fig. 8. a) The simplified equivalent small signal circuit of a single-stage BJT amplifier; b) The

Thévenin equivalent circuit; c) The port's characteristic curve, indicating the linearity; d) Circuit used to calculate the parameters $V_{T h}=V_{o c}$ and $R_{e q}$; e) Connection to terminals A, B of the $R_{d u} 7$ voltage-controlled (vc) nonlinear resistor. 
Analyzing, by the ACAP program, [3], the circuit in Fig. 8, e results the following values for the voltage and current of the nonlinear resistor $R_{d u}$, $U_{7}=0.84 \mathrm{~V}$ and $I_{7}=0.84 \mathrm{~mA}$. If we analyze, with the same ACAP program, [3], the circuit from Figs. 9, a and $9, \mathrm{~b}$ we obtain the results identical to the above $U_{3}=0.84 \mathrm{~V}$ and $I_{3}=0.84 \mathrm{~mA}$.

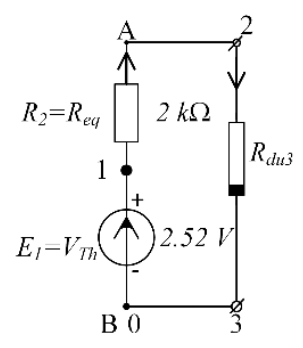

(a)

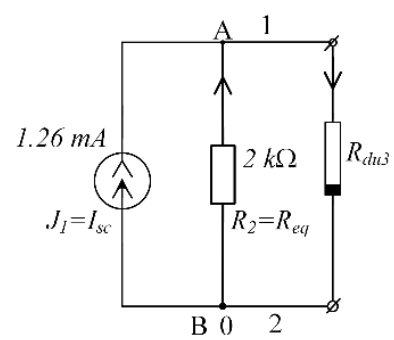

(b)
Fig. 9. a) Thevenin equivalent circuit; b) Norton equivalent circuit.

Simplicity of operation, port representation using either the Thévenin or Norton equivalent circuits involves a degree of stiffness. As seen in Fig. 8, c, the Thevenin or Norton models each occupy a single point on the characteristic line at the place where the line intersects one of the axes.

The limitation for Thévenin or Norton model is that it represents only the "current supply" network with no information given about the "target" network, unless the two are connected and the analysis is done with the combined circuit. This is true for a lot of circuit applications, when all that is required is a simple two-terminal linear circuit linked to the target circuit for the remainder of the process; however, we execute the analysis only when the two are coupled. While the circuit complexity developed in this manner may not be immediately apparent for a single port connection, it may become rather considerable when numerous ports are connected.

Other instances exist when circuits on both sides of a port must participate in a (sources or components) exchange; in these instances, a more dynamic port modeling approach may be required. There are several examples in source transformation, noise source modeling, and power transfer. Port cancelling is another example that uses Hybrid modelling, as discussed in the other paper.

\section{Conclusions}

The paper presents the necessary and sufficient conditions that must be met by one-port linear circuits to be replaced by equivalent Thévenin and Norton circuits. These circuits are widely used in the analysis of analog circuits. The Thévenin and
Norton theorems are simply proved based on the superposition theorem.

The main value of Thévenin's theorem, as well as Norton's theorem, is that it allows us to replace any part a circuit which forms a linear resistive oneport (but which is of no interest in each situation) by only two circuit elements without affecting the solution of the solution of the remainder of the circuit.

In this paper the equivalent Thévenin and Norton circuits are correctly defined. These circuits allow the separation of the linear portions of the electronic circuits from the non-linear ones and in this way the polarization process of the electronic devices becomes much more efficient. When the analysis of linear and/or nonlinear circuits have a small number of nonlinear circuit elements, their analysis, synthesis, and simulation are performed much more efficiently if the nonlinear part of the circuit is separated from the linear one. This separation can be done very easily using the equivalent Thevenin and Norton circuits.

By determining the equivalent Thévenin or Norton circuits in relation to the load connection terminals of a system of two or more magnetic coupled coils, used in the construction of wireless power transfer systems, optimal load parameters are determined so that the active power transmitted to the load is maximum.

A major difficulty in approaching the analog circuits is the DC polarization - obtaining the desired operating points by (through) a fast convergence procedure; and the problem worsens due to the increase size and complexity of the circuit. The analysis can even lead to multiple cc operating points, or to the instability of these operating points due to positive reactions.

\section{REFERENCES}

[1]. Esteban Tlelo-Cuautle edit., Advances in analog circuits, January 2011,

https://www.researchgate.net/publication/23400797.

[2]. Mourad Fakhfakh, Marian Pierzchala, Editors, Pathological elements in analog circuit design, Springer U.S., 2018, ISBN 978-3-319-75157-3; 9783-319-75156-6, ISSN

1876-1100, WOS:000441385600014.

[3]. M. Iordache, Symbolic, numeric-symbolic, numeric simulation of the analoque circuits - User's Guides, MATRIX ROM Publishing, Bucharest, ISBN $978-$ $606-25-0126-6,621.3 .049 .77$.

[4]. T.L. Pillage, R.A. Rohrer, and C. Visweswariah, "Electronic circuit \& system simulation methods," New York, McGraw-Hill, 1995.

[5]. J. Vlach and K. Singhal, Computer Methods for Circuit Analysis and Design, Van Nostrand Reinhold Electrical/Computer Science and Engineering Series, 1983. 
[6]. L.W. Nagel, "SPICE2, A Computer Program to Simulate Semiconductor Circuits," Univ. of California, Berkeley, CA, Memorandum no. ERLM520, 1975.

[7]. Mike Smith, "WinSpice3 User's Manual, v1.05.08", http://www.ousetech.co.uk/winspice2/, May 2006.

[8]. C.W. Ho, A.E. Ruehli, and P.A.Brennan, "The modified nodal approach to network analysis," IEEE Trans. Circuits Syst., vol. CAS-22, no.6, pp.504-509, June 1975.

[9]. C. A. Desoer and E. S. Kuh, Basic Circuit Theory. New York: McGraw Hill, 1969.

[10]. Y. Inouea, "Dc analysis of nonlinear circuits using solution-tracing circuits," Trans. IEICE (A). vol. J74 A, pp. 1647-1655, 1991.

[11]. L. B. Goldgeisser and M. M. Green "A Method for Automatically Finding Multiple Operating Points in Nonlinear Circuits," IEEE Trans. Circuits Syst. I, vol. 52, no. 4, pp. 776-784, April. 2005.

[12]. R. C. Melville, L. Trajkovic, S.C. Fang, and L. T. Watson, "Artificial parameter homotopy methods for the dc OP problem," IEEE Trans. Computer-Aided Design, vol. 12, no. 6, pp. 861-877, Jun. 1993.

[13]. A.S. Sedra, and K.C. Smith, Microelectronic Circuit 6th ed. Oxford University Press, 2010.

[14]. R. Jacob. Baker, CMOS, Circuit Design, Layout, and Simulation, 2nd ed. IEEE Press, Wiley

[15]. Interscience, 2008, pp. 613 - 823.

[16]. R. Hashemian, "Designing Analog Circuits with Reduced Biasing Power", to be published in the Proceedings of the 13th IEEE International Conf. on Electronics, Circuits and Sys., Nice, France Dec. 1013, 2006.

[17]. R.C.Jaeger, and T.N. Blalock, Microelectronic Circuit Design 4th ed. Mc Graw-Hill Higher Education, 2010.

[18]. M. Iordache, Lucia Dumitriu, Computer-Aided Simulation of Analog Circuits - Algorithms and Computational Techniques, Editura POLITEHNICA Press, Bucureşti 2014, ISBN 978-606-515-567-1, Vol. Al II-lea - ISBN 978-606-515-586-2 